\title{
SPONTANEOUS CORONARY ARTERY DISSECTION
}

Singapore Med J 2017; 58(2): 111-112 doi: 10.11622/smedj.2017008

Dear Sir,

A 44-year-old man with no known risk factors for coronary artery disease presented with a sudden onset of crushing chest pain after an $85-\mathrm{km}$ bicycle ride. On admission, the patient's electrocardiogram showed sinus rhythm and his troponin I level was $1.53 \mathrm{ng} / \mathrm{mL}$, which increased to $15 \mathrm{ng} / \mathrm{mL}$ after six hours, following medication. Cardiovascular examination was normal with no stigmata of connective tissue disorders. The patient was treated with aspirin, ticagrelor, enoxaparin and atorvastatin, but developed an ongoing chest discomfort. Coronary angiography revealed a linear intimal tear involving the left anterior descending artery (LAD), with associated TIMI (Thrombolysis in Myocardial Infarction) 2 flow (Figs. 1a \& b). The patient's other coronary arteries revealed no atherosclerotic disease and his ST segments remained normal. However, the chest pain persisted and therefore, a bare metal stent was deployed (Fig. 1c).

Primary spontaneous coronary artery dissection (SCAD) remains a rare presentation of acute coronary syndrome, with a prevalence of around $0.1 \%-0.4 \%{ }^{(1)}$ Dissection of the coronary intima or media leads to the formation of a false lumen. This false lumen may expand and propagate, obstructing flow within the true lumen, which may produce symptoms of myocardial ischaemia. Women who are in gestation or the peripartum period, with no classical cardiovascular risk factors, appear to have the highest risk. ${ }^{(2)}$ It has been hypothesised that the aetiology of SCAD in women is hormonal balance and appropriate pregnancy-related changes to cardiovascular physiology. ${ }^{(3)}$ In contrast, SCAD in men is principally preceded by substance abuse or extreme physical activity. The latter is thought to increase shear stress, leading to an intimal tear, which was the case in our patient. Recent studies have found a strong association between fibromuscular dysplasia and SCAD; however, the true prevalence remains unknown. ${ }^{(4)}$ Multiple arteries are dissected in around $20 \%$ of cases and the LAD is the most common vessel affected. ${ }^{(5)}$

The optimal therapy for SCAD has yet to be determined and single-centred retrospective cohort studies are still our best source of evidence. ${ }^{(5,6)}$ Conservative management in an otherwise stable patient with normal distal flow is now advocated as the management strategy of choice; follow-up angiography has shown complete resolution for such patients. ${ }^{(5)}$ Primary coronary intervention (PCI) has a potentially critical role, but appropriate selection is required based on the presence of ischaemia or infarction. Technical failure is common due to the passage of the wire into the false lumen and loss of coronary blood flow from the propagation of dissection following stent placement. Optical coherence tomography can provide anatomical evaluation for atherosclerotic disease when diagnosis is ambiguous. In addition, complications can be reduced by confirming guide-wire placement in the distal true lumen and optimising stent deployment. ${ }^{(2)}$ Coronary artery bypass grafting has demonstrated high rates of late bypass graft occlusion. ${ }^{(5)}$ This may reflect recurrent dissection in the native coronary arteries or competitive flow. Those who survive the acute episode have a favourable outcome; the ten-year SCAD recurrence rate is $29.4 \% .^{(5)}$
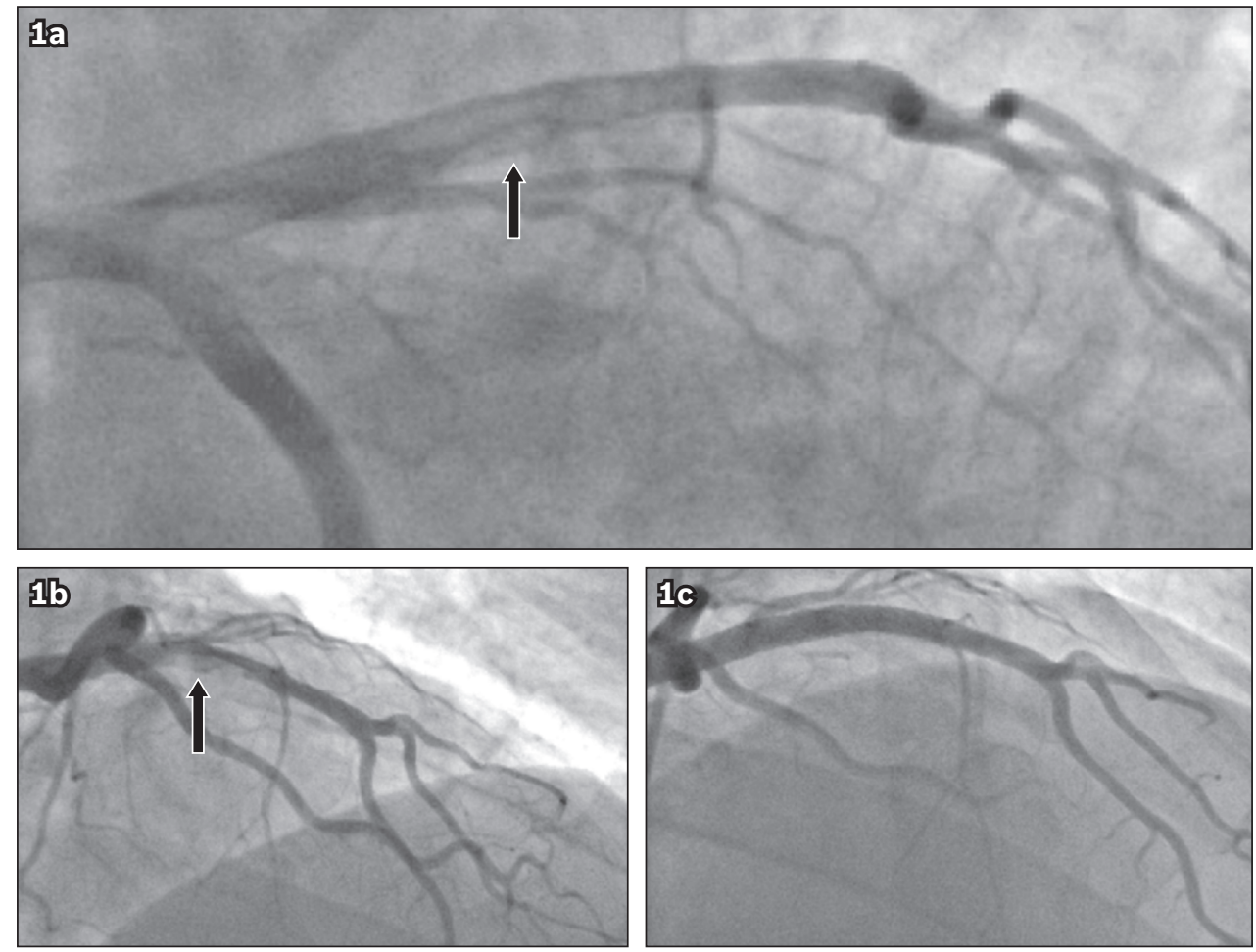

Fig. 1 Coronary artery dissection of the left anterior descending artery (LAD) and resolution. Coronary angiograms show (a \& b) the right anterior oblique view of the dissection flap (arrows); and (c) the LAD following the deployment of a bare metal stent. 
In the case of our patient, $\mathrm{PCl}$ was performed and appeared effective, although the patient remained under close surveillance. SCAD is a rare condition, but it must be considered in the differential diagnosis of acute myocardial infarction in younger patients with no cardiac risk factors.

\section{Yours sincerely,}

Alexander Dashwood ${ }^{1}, 2$, Selvanayagam $\underline{\text { Niranjan }}^{1,2}$, Saheb $\underline{\text { Al-Daher }}^{1}$, Jerome Goldstein ${ }^{1}$

${ }^{1}$ Cardiology Department, Gold Coast University Hospital, ${ }^{2}$ Griffith University School of Medicine, Queensland, Australia. icuris247@googlemail.com

\section{REFERENCES}

1. Rashid HN, Wong DT, Wijesekera H, et al. Incidence and characterisation of spontaneous coronary artery dissection as a cause of acute coronary syndrome-a single-centre Australian experience. Int J Cardiol 2016; 202:336-8.

2. Alfonso F. Spontaneous coronary artery dissection: new insights from the tip of the iceberg? Circulation 2012; 126:667-70.

3. Sheikh AS, O'Sullivan M. Pregnancy-related spontaneous coronary artery dissection: two case reports and a comprehensive review of literature. Heart Views 2012; 13:53-65.

4. Saw J, Ricci D, Starovoytov A, Fox R, Buller CE. Spontaneous coronary artery dissection: prevalence of predisposing conditions including fibromuscular dysplasia in a tertiary center cohort. JACC Cardiovasc Interv 2013; 6:44-52.

5. Tweet MS, Hayes SN, Pitta SR, et al. Clinical features, management, and prognosis of spontaneous coronary artery dissection. Circulation 2012; $126: 579-88$.

6. Vanzetto G, Berger-Coz E, Barone-Rochette G, et al. Prevalence, therapeutic management and medium-term prognosis of spontaneous coronary artery dissection: results from a database of 11,605 patients. Eur J Cardiothorac Surg 2009; 35:250-4. 